\title{
Nurses' awareness of ototoxic pre-operative cleaning solution in ENT surgery
}

PY Wong ${ }^{1}$, H Zhang ${ }^{1}$, J Thaj ${ }^{2}$, S G Kumar

${ }^{1}$ Department of Ear, Nose and Throat Surgery, Queens Hospital, Romford, UK

${ }^{2}$ Department of Ear, Nose and Throat Surgery, Royal National Throat, Nose and Ear Hospital, London, UK

\section{Objective:}

Alcohol and isopopryl alcohol solutions are both ototoxic 1,2. A case series showed 13 patients sustained total cochleo-vestibular loss following the use of $0.5 \%$ chlorhexidine and $70 \%$ isopropyl alcohol solution in myringoplasties ${ }^{3}$. We aim to assess ENT nurses' awareness of the types of preoperative cleaning solution and their contents.
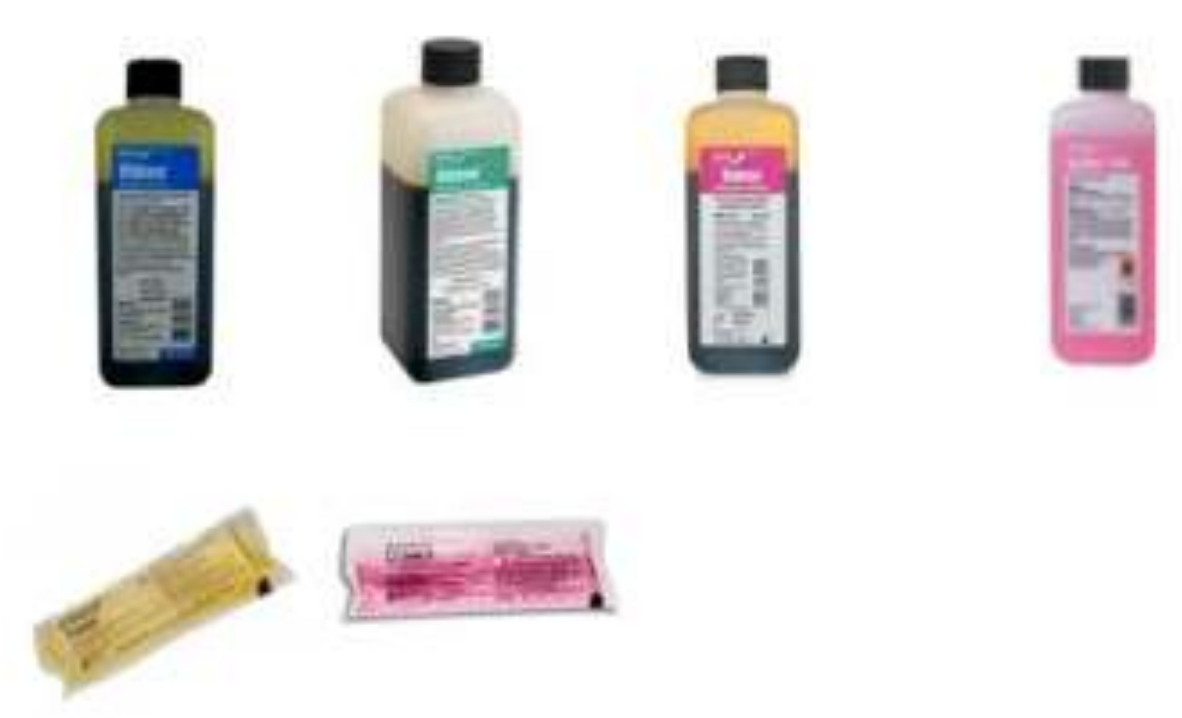

\section{Method}

We surveyed 27 ENT nurses in two ENT departments between February-October 2017 using questionnaires. The average experience of these ENT nurses was 11 years.

\section{Results}

$70 \%$ of nurses do not know the contents of cleaning solution and its potential ototoxicity, believe there is lack of training in the use of cleaning solution and feel it is the responsibility of both surgeons and nurses to ensure the correct solution is used.

This survey highlighted the issues of shared theatre with other surgical specialties which may require alcohol cleaning solution and the general lack of awareness among surgical and scrub team.

\section{Conclusion}

It is important that surgeons check the cleaning solution prior to use. We propose an ENT UK guidance on the ototoxicity of pre-operative cleaning solution. 